\title{
A severe recessive and a mild dominant form of Charcot-Marie- Tooth disease associated with a newly identified Glu222Lys GDAP1 gene mutation
}

\author{
Dagmara Kabzińska1, Katarzyna Kotruchow1, Joanna Cegielska1,2, Irena Hausmanowa- \\ Petrusewicz ${ }^{1}$ and Andrzej Kochański ${ }^{1 凶}$
}

${ }^{1}$ Neuromuscular Unit, Mossakowski Medical Research Centre, Polish Academy of Sciences, Warsaw, Poland; 2llnd Department of Neurology, Warsaw Medical University, Poland

\begin{abstract}
Charcot-Marie-Tooth (CMT) disease caused by mutations in the GDAP1 gene has been shown to be inherited via traits that may be either autosomal recessive (in the majority of cases) [CMT4A] or autosomal dominant [CMT2K]. CMT4A disease is characterized by an early onset, and a severe clinical course often leading to a loss of ambulation, whereas CMT2K is characterized by a mild clinical course of benign axonal neuropathy beginning even in the 6 th decade of life. Clinical data from a GDAP1 mutated patient suggests that the presence of a particular mutation is associated with a certain trait of inheritance. The association of a particular GDAP1 gene mutation and a dominant or recessive trait of inheritance is of special importance for genetic counseling and the prenatal diagnostics as regards severe forms of CMT. In the present study we report on two CMT families in which a newly identified Glu222Lys mutation within the GDAP1 gene segregates both in autosomal dominant and recessive traits. Our study shows that at least some GDAP1 gene mutations may segregate with the CMT phenotype as both dominant and recessive traits. Thus, genetic counseling for CMT4A/CMT2K families requires more extensive data on GDAP1 phenotype-genotype correlations.
\end{abstract}

Key words: GDAP1, Charcot-Marie-Tooth disease, autosomal dominant and recessive traits

Received: 12 December, 2013; revised: 12 July, 2014; accepted: 17 July, 2014; available on-line: 22 October, 2014

\section{INTRODUCTION}

Over hundreds of years genetic counseling in Charcot-Marie-Tooth disorders was hampered by genetic heterogeneity in this group, ensuring that the same phenotype of hereditary peripheral neuropathy may be associated with different mutations of numerous genes. Neither morphological nor electrophysiological studies in CMT proved being able to correlate a particular form of CMT with a certain trait of inheritance. However, recent achievements in the field of molecular diagnostics, which extend also into the CMT area, have opened the door to genetic counseling based on the identification of a certain mutation transmitted as X-linked, autosomal dominant or recessive traits (Ionasescu, 1995). Additionally, for CMT forms with a severe clinical course the findings of molecular genetics are of special importance where prenatal diagnostics are being concerned.

In 2002, two independent groups identified mutations in the GDAP1 gene responsible for the severe form of Charcot-Marie-Tooth disease with an autosomal recessive trait (CMT4A) (Baxter et al., 2002; Cuesta et al., 2002). Within 10 years over 100 patients harboring mutations in the GDAP1 gene have been reported all over the world (Cassareau et al., 2011). In 2005 Claramunt and colleagues reported on the Arg120Trp mutation in the GDAP1 gene inherited as an autosomal dominant trait (Claramunt et al., 2005). The Arg120Trp mutation segregates with a phenotype of a mild axonal neuropathy with a wide spectrum of clinical features (Siviera et al., 2010). In contrast to GDAP1 gene mutations inherited as an autosomal recessive trait, the heterozygous dominant mutations of this gene are reported to be of variable penetrance. Some dominant GDAP1 gene mutations were even found in healthy individuals in the 7-th decade of life. Interestingly, in some CMT-affected patients, dominant GDAP1 gene mutations segregate with asymmetrical mononeuropathy limited to the lower limbs (Zimon et al., 2011).

Little is known of the molecular basis for the recessive and dominant inheritance of GDAP 1 gene mutations. The effect of the GDAP1 mutations (amino-acid substitutions) is strongly dependent on the mode of inheritance. In general, recessively-inherited mutations in the GDAP1 gene result in reduced fission activities with a predominance of tubular and mixed (tubular and vesicular) mitochondria (Niemann et al., 2005). In turn, mutations transmitted as a dominant trait have a negative influence on mitochondrial fusion, as with the mutations of another CMT gene - MFN2. Excessive mitochondrial fragmentation also increases the susceptibility to apoptosis. Moreover, dominantly-inherited mutations influence ROS levels and transmembrane potential (Niemann et al., 2009). Mutations leading to GDAP1 protein truncation constitute a separate group of mutations resulting in disturbance of GDAP1 anchoring to the mitochondrial outer membrane, and in consequence to alterations in mitochondrial dynamics (Niemann et al., 2005).

To the best of our knowledge, we report here for the first time the newly-identified Glu222Lys mutation in the

e-mail: akochanski@imdik.pan.pl Abbreviations: CMT, Charcot-Marie-Tooth 
GDAP1 gene segregating in both autosomal dominant and recessive traits.

\section{CLINICAL REPORT}

Family A. The parents of the proband originate in the eastern part of Poland, from a family without apparent consanguinity. The 46-year-old father (II-3) of the patients is healthy and no abnormalities were found in him on neurological examination. The 44-year-old mother (II-4) considers herself healthy, though neurological examination confirmed the absence of ankle jerks (Fig. 1).

III-2-20 years old. This female patient was born following a normal, uneventful pregnancy. Parturition was provoked, and the baby was born at weight $2600 \mathrm{~g}$ and length $49 \mathrm{~cm}$. An APGAR score of 10 points was given. The infant sat at 6 months and started to walk at 11 months. At age 2, her clumsy gait on a broad base with foot dropping was noted by her parents, who noted that she often fell down. At the age of 3 years she underwent surgery for a plano-valgus foot. At the age of 3 years 10 months, she was first hospitalized in the Neurological Department and the diagnosis of CMT2 was established. At the age of $310 / 12$ months she had wasting of the calves, contraction of the Achilles tendons, weak ankle and knee reflexes, though preserved deep tendon reflexes in the upper limbs. ENG analysis at this age showed massive sensory neuropathy (no recordable amplitudes from the sensory fibers of median and sural nerves) and reduction of the CMAP amplitudes in the median and tibial nerves ( 3.5 and $0.027 \mathrm{mV}$, respectively). Other laboratory tests (for glucose, CPK, lactic acid, pyruvic acid, AspAT, ALAT, cerebrospinal fluid) gave results within the normal range.

At the age of 19 years III-2 was hospitalized again. She was an active intelligent young woman achieving good results at school. Her height was low, at $152 \mathrm{~cm}$, and her gait was very clumsy with foot dropping. She presented with flaccid paresis of the lower limbs, and had severe wasting of the muscles of the upper and lower limbs (Fig. 2 hands III-2). Pes cavus deformity was observed.

III-3-17 years old. This patient born from a second, normal, uneventful pregnancy with a weight of $3100 \mathrm{~g}$ and an APGAR score of 10 is the brother of the proband (III-2). Following the diagnosis of CMT in the sister, he was first examined at the ages of 4 and 6 months, though no abnormalities in neurological examination were found at that stage. However, at the age of 16 months his gait was clumsy, with walking on the broad base and frequent falling down. The results of other laboratory tests were within the normal range. At the age of 17 years, he presented with foot-dropping gait, generalized wasting of the distal muscle that also involved the small muscles of the hand (Fig. 2 - hands of III-3). He had profound abnormalities in the sensory modalities.

Family B. III-10 is a patient aged 73 years who had observed his gait becoming clumsy when aged 68. Neurological examination when he was 72 revealed that the left ankle jerk was present, though the right was absent, as were the knee reflexes. No other abnormalities were found in the patient.

In ENG examination, the CMAP amplitudes in the peroneal and tibial nerves were extremely reduced (to 0.1 $\mathrm{mV}$ ), and there was no response from the sensory fibers of most examined nerves, except for a residual sensory response of the radial one (A $3 \mathrm{uV}, \mathrm{CV} 40 \mathrm{~m} / \mathrm{sec}$ ).

In the sister of the patient, III-12, aged 71 years, nothing more than diminished ankle reflexes were found. Back pain was revealed by anamnesis. Causes of disease were seen in osteoarthritis of the spine as detected by MR imaging. The right ulnar, median, right and left tibial, peroneal and sural nerves were examined, no abnormalities being found in either motor or sensory fibers of the examined nerves, except as regards the CMAP amplitude of the left tibial nerve, which was reduced to 3.0 $\mathrm{mV}$ (normal $>5.0 \mathrm{mV}$ ). The latency to $\mathrm{F}$ wave from this nerve was prolonged $(57.7 \mathrm{~ms})$. F-wave latency was also slightly prolonged in the right peroneal and right tibial nerves.

In addition, EMG examination performed on the patient revealed a chronic and moderate neurogenic pattern in the muscles of the left lower limb (L5-S1). However, no abnormalities were detected in the arrangement of the motor units in other muscles of the upper and lower limbs.

\section{METHODS}

We recruited 20 CMT2 Polish families consulted at the Neuromuscular Unit, Mossakowski Medical Research Centre of the Polish Academy of Sciences, and the Department of Neurology of Warsaw Medical University. Clinical and genetic study gained the approval of the local Medical and Ethics Committee at the Medical University of Warsaw. All examined patients and their family members or their legal representatives signed an informed consent form in respect of the DNA analysis.

Genomic DNA was isolated from white blood cells of the CMT patients and their family members. As a standard procedure, duplication or deletion of the PMP22 gene was excluded using the real time PCR (Q-PCR) approach (Aarskog, Vedeler, 2000) in III-10, III-3 oraz III2. All coding exons of the GDAP1 gene were sequenced directly with primers previously reported (Cuesta et al., 2002). Sequencing data were analyzed by comparing with a reference mRNA isoform sequence NM_018972.2 and genomic sequence NG_008787.1 of the GDAP1 gene.

We also performed analysis of mitochondrial DNA integrity (mtDNA). Recently, Rouzier and coworkers demonstrated that in the patients with optic nerve atrophy, mitochondrial DNA fragmentation results from a novel missense mutation in the MFN2 gene (Rouzier et al. 2011). We isolated whole DNA from the leukocytes from peripheral blood, and amplified the whole mitochondrial genome as well as the nuclear HPRT gene as a control using an Expand High Fidelity PCR System (Roche) and the primers described previously (Rouzier et al., 2011).

Short Tandem Repeat analysis. To test the hypothesis of a common ancestor for two CMT families originating in the same region of Poland, haplotype analysis was performed with 6 highly-informative short tandem repeat microsatellite (STR) markers (D8S279, D8S548, D8S551, D8S286, D8S1144, P26ACG) located in 8q13q21 region. STRs were first PCR-amplified with fluorescently (FAM) labeled primers (sequences are available at www.ncbi.nlm.nih.gov); fragments were combined with formamide and GeneScan'TM $500 \mathrm{Liz}^{\circledR}$ Size Standard (Life Technologies) and size-separated on ABI3130xl DNA Analyzer. Genotyping results were assessed using Peak Scanner ${ }^{\mathrm{TM}}$ Software v1.0 (Life Technologies). 

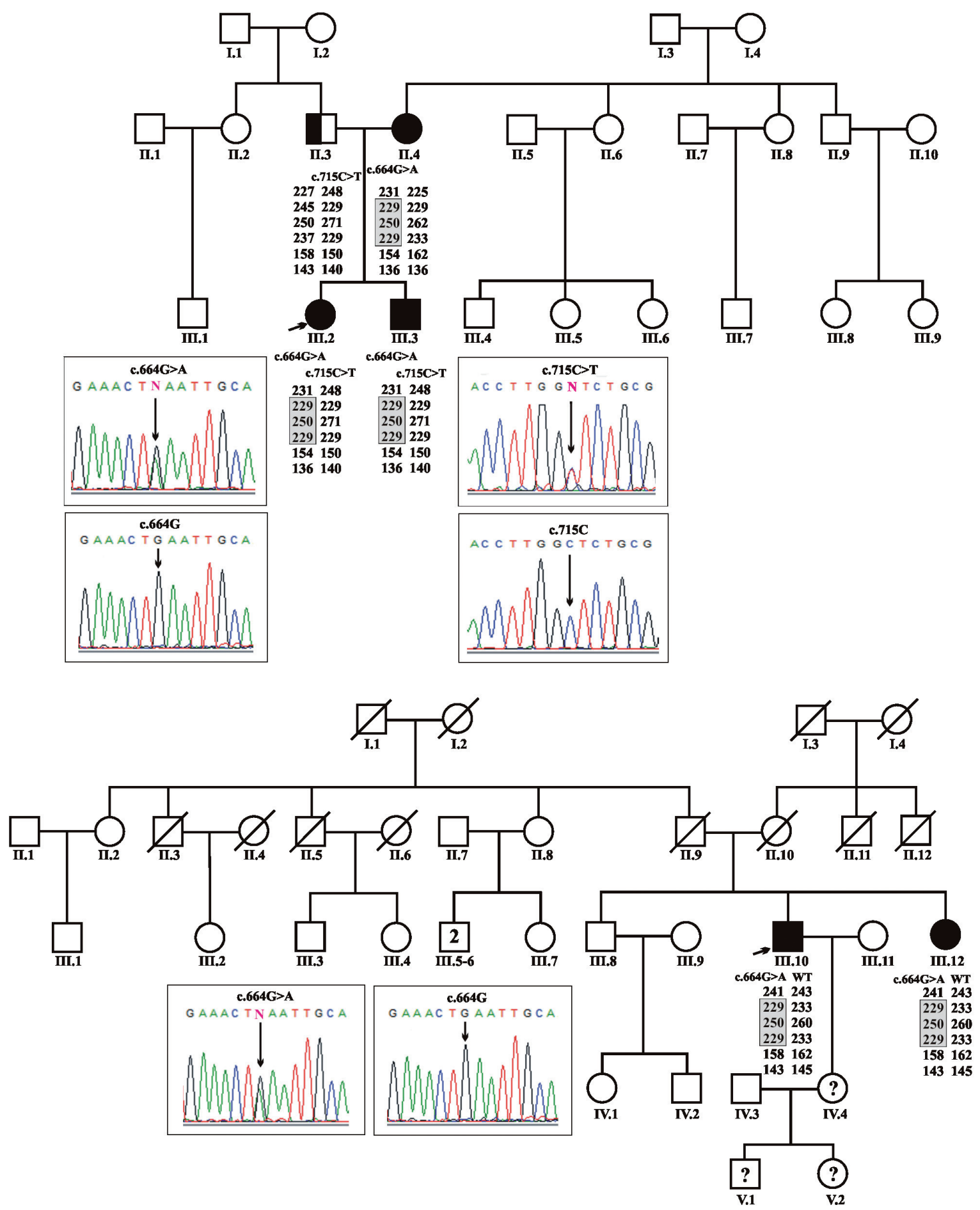

Figure 1. Pedigrees of two Polish families, A (upper panel) and B (lower panel), affected by CMT4A and CMT2K disease.

Filled circles or squares represent individuals diagnosed with CMT, empty symbols unaffected family members, and the filled gray bar below the symbols the disease-associated haplotype. The latter (gray box) are identical in two families, indicating possible common heritage. The chromatograms below the pedigrees show both the mutated and wild-type GDAP1 gene allele.

\section{RESULTS}

In the families recruited to this study, diagnosis of Charcot-Marie-Tooth type 2 (CMT2) disease was established. CMT2 was diagnosed on the basis of clinical evaluation and nerve conduction studies. The inclusion criterion for this study was axonal-type pe- ripheral neuropathy diagnosed on the basis of $\mathrm{ENG}$ findings.

The reference values for dosage of the PMP22 gene in healthy individuals of our study range between 0.700 and 1.090 (Kabzinska et al., 2009). The values for the dosage of the PMP22 gene in the DNA samples from examined individuals ranged from 0.926 to 1.028 . 


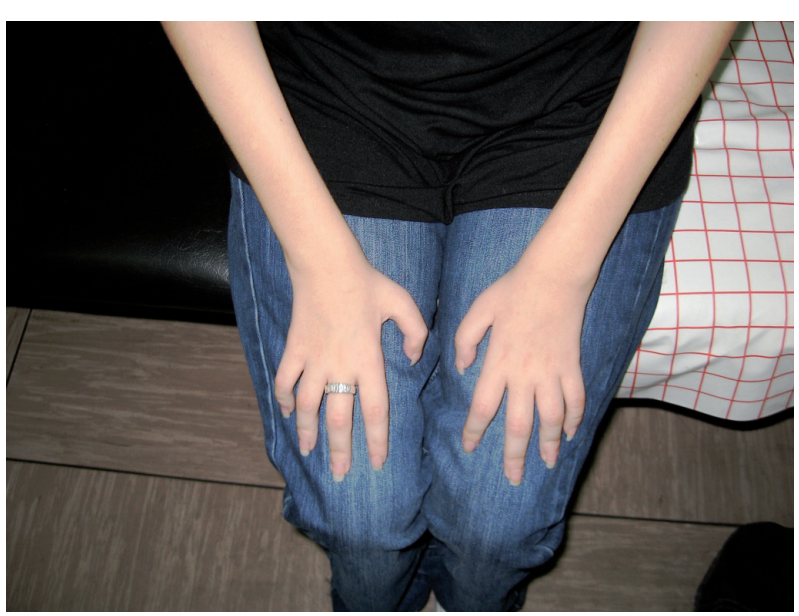

Figure 2. In the proband (III-2), aged 17 severe wasting of the distal upper-limb muscles and small hand muscles is observed.

Direct sequence analysis of the six exons of the $G D A P 1$ gene referred to revealed a new heterozygous c. $664 \mathrm{G}>\mathrm{A}$ mutation in the two investigated families. In family $B$ the mutation occurred in the proband III-10, and his sister, III-12. The same sequence variant was found in family A in II-3 (heterozygote) and in a compound heterozygous state with c.715C $>\mathrm{T}$ (p.Leu239Phe) mutation in III-2 and III-3. The healthy mother II-4 of the proband was a carrier of c. $715 \mathrm{C}>\mathrm{T}$ mutation. The c.664G $>$ A mutation results in the substitution of a glutamine by lysine at codon 222 (p.Glu222Lys), located in the C-terminal homologous to the glutathione S-transferase (GST-C) domain of the GDAP1 protein.

We did not observe any fragmentation of $\mathrm{mt}$ DNA in our patients, in the analysis of DNA integrity.

\section{DISCUSSION}

A question concerning the pathogenic effect of mutations in the GDAP1 gene raises issues of special importance in the context of prenatal and preimplantation diagnosis in the CMT4A-affected families. In fact, in contrast to the majority of CMT forms, CMT4A is a severely disabling disease resulting in a loss of ambulance even in young patients beginning their second decade of life. Phenotypically dominant and recessive GDAP1 gene mutations represent extreme points on the scale of clinical severity. CMT2K disease caused by a heterozygous GDAP1 gene mutation represents a rather mild form of peripheral neuropathy starting in adults or old patients and characterized by a benign clinical course, or even remaining symptomatic only on the subclinical level (subtle EMG abnormalities). To date, GDAP1 gene mutations have also been classified as dominant and recessive. A trait of inheritance was treated as a constant feature of a certain mutation. Thus the Leu239Phe mutation reported in numerous European CMT families was shown to be inherited as an autosomal recessive trait in both homozygous and compound heterozygous configurations (Kabzinska et al., 2010). The Arg120Trp mutation in the GDAP1 gene segregates with the prevailing form of CMT disease with a wide clinical spectrum (Sivera et al. 2010). Interestingly, in our study, we detected the new Glu222Asp mutation in three configurations: (i) a heterozygous mutation in a 70 -year-old clinically asymptomatic woman with benign ENG abnormalities whose acquired origin is hard to preclude, (ii) a heterozygous dominant mutation in a 74-year-old patient with a 4-year history of disease and evident electrophysiological abnormalities, (iii) in a 40-year-old woman with CMT phenotype limited to the loss of ankle reflexes, and (iv) a heterozygous recessive mutation with Leu239Phe manifesting as a severe CMT disease in two patients.

Although our study reveals a quite wide spectrum of clinical features we cannot definitively preclude the GDAP1 gene mutation reported by us resulting in other forms of CMT. Our study shows that at least for some mutations of the GDAP1 gene a trait of inheritance should not be established on the basis of data for a single family. Given the reduced penetrance of Glu222Asp mutation observed in patients (III-10 and III-12 in family B), a severe phenotype in patients harboring Leu239Phe/Glu222Asp mutations is surprising. In fact, our study confirms that the final effect of two mutations cannot be anticipated as the sum of two different gene alterations i.e. a strong and weak mutation. In the initial studies dedicated to GDAP1 mutations, missense substitutions were thought to be associated with a mild CMT phenotype, whereas nonsense mutations led to a severe phenotype (Niemann et al., 2009). Recently, we reported on Leu239Asp homozygous mutation segregating with an extremely severe phenotype of CMT. In fact, despite its missense character, Leu239Asp mutation results in disturbed targeting of the mutated GDAP1 protein into the mitochondrial outer membrane. A missense mutation was thus shown to act as nonsense mutation of the GDAP1 gene at the cellular level (Vital et al., 2012).

To test for the cellular effects of the Glu222Asp mutation, we hypothesized its impact on the integrity of the mitochondrial DNA molecule. Recently, Pro45Lys mutation within the Mitofusin 2 gene responsible for maintaining the mitochondrial network was shown to result in the fragmentation of mitochondrial DNA (Rouzier et al., 2012).

The GDAP1 protein also contributes to mitochondrial network dynamics and, together with the whole protein apparatus, e.g. mitofusin 2, coordinates the processes of fission and fusion of these organelles. We postulate that the mutations found in the GDAP1 gene in the probands and their families could affect, and cause microdeletions in, mtDNA. In this study we have shown that, in contrast with Pro45Lys mutation in the $M f n 2$ gene resulting in the fragmentation of mitochondrial DNA, Glu222Lys mutation does not act via a molecular mechanism leading to the fragmentation of mitochondrial DNA.

We cannot preclude Glu222Asp mutation acting via another mechanism not associated with the integrity of mitochondrial DNA. Equally, as material from patients other than blood (e.g. skin fibroblasts, muscle or nerve biopsy) was not available, aberrations in the quality of mitochondrial DNA in the direct regions of clinical manifestation of the disease cannot be ruled out.

A cumulative effect of two weak mutations in GDAP1 and Mfn2 genes (Arg120Trp and Val160fsx26 respectively) has been documented recently in one French CMT family. The parents of the proband - who is a compound heterozygote for GDAP1/Mfn2 mutations - were asymptomatic (father) or displayed a very mild CMT2 clinical course (mother - Arg120Trp mutation in the GDAP1 gene). Interestingly, their daughter manifested with a severe form of CMT2 disease (Vital et al. 2012). Ammar and colleagues reported a Belgian CMT family in which compound heterozygosity for Arg120Trp and Gly271Arg GDAP1 gene resulted in a severe phenotype of two wheelchair dependent siblings aged 12 and 20 years, respectively (Ammar et al., 2003). 
The heterozygous Glu222Lys mutation was observed only in three individuals. It seems on this basis that this mutation has stable penetrance, resulting in a late-onset mild form of axonal neuropathy. As with previous observations reporting an asymmetrical pattern of Electroneurography (ENG) abnormalities in patients harboring dominant mutations within the GDAP1 gene, patient III-12. manifested with isolated reduction of CMAP amplitude in the left tibial nerve. However, given the age of the patient (71) we could not definitively exclude abnormalities having been acquired in this patient.

Given the results of the ENG and EMG studies performed on III-12 and MRI findings from the L-S portion of the spine, we established a diagnosis of leftside radiculopathy. Although the neuropathy found is generally an acquired type, it remains possible that the Glu222Lys mutation found in the patient did not contribute to phenotype.

Among GDAP1 gene mutations transmitted via an autosomal dominant trait was Arg120Trp mutation characterized in a group of patients. This showed variable penetrance, as in some individuals there was no abnormality on neurological and electrophysiological examination even in old patients (Sivera et al., 2010). Recently, a three-generation CMT family from Italy with Arg120Gly mutation was described. Electrophysiological findings encompassing 7 members of this family revealed a marked variability to an asymmetrical process. Interestingly, the oldest patient aged 79 years showed only the reduced values for sensory action potentials (SAPs) in the sural and peroneal superficial nerves (Ammar et al., 2003). In the Finnish CMT2 family with His123Arg heterozygous mutation in the GDAP1 gene, four individuals in the first generation of the family were seen to be asymptomatic carriers (Zimon et al., 2011).

We may conclude that Glu222Lys mutation in the GDAP1 gene reported by us may result in a late-onset CMT2 disease with a mild clinical course and variable penetrance in a heterozygous state. In a compound heterozygosity with another GDAP1 recessive mutation, Glu222Lys mutation leads to a severe, early-onset CMT2 disease. Further studies are needed to establish the range of penetrance of Glu222Lys mutation in both dominant and recessive traits of inheritance. In the previous study reporting CMT families with dominant GDAP1 gene mutations, we documented cardiac arrhythmia cosegregating with disease in one Polish family (Zimon et al., 2011). In one patient reported here conduction disturbances localized over the right heart ventricle were also found. We cannot exclude the coexistence of GDAP1 gene mutations with heart arrhythmia being incidental, though GDAP1 protein is located in the mitochondrial outer membrane. In fact, mutations of the mitochondrial proteins may often be associated with the heart failure.

Glu222Lys mutation is located in the C-terminal domain of GDAP1 protein, homologous with glutathione S-transferase domains (GST-N and GST-C).

In the previous study we demonstrated a founder effect for Leu239Phe mutation in the GDAP1 gene, at least in the East European population (Kabzinska et al., 2010). In this study we have also analyzed 8 q11.4 region with a set of STR markers (Fig. 1). As in the previous study we confirmed the presence of a common haplotype for carriers of Leu239Phe mutation, supporting our previous hypothesis concerning a founder effect for this mutation, at least in the case of the East European population. As regards Glu222Lys mutation in GDAP1 gene, we are not able to demonstrate an evident founder effect for the mutation. Thus, we can only speculate that carriers of Glu222Lys mutation could have a common ancestor. To conclude, there is a question as to whether a third class of GDAP1 gene mutations should be added to the existing recessive and dominant variants A second, clinically important question concerns the spectrum of phenotypes associated with dominant GDAP1 gene mutations. Interestingly, within 12 years since its first description in consanguineous Algerian families, CMT4A evolved to its mild form of CMT2 disease. Finally, some GDAP1 gene mutations characterized by extremely variable penetrance may manifest as both autosomal recessive and dominant traits. Moreover, the Glu222Lys mutation in the GDAP1 gene shows a variable penetration which cannot be related to the age of the patient.

\section{Acknowledgements}

This study was supported by a grant from the Polish National Science Centre No. 2012/B/N24/01748 to D.K. The authors thanks the patients for excellent cooperation during this study.

\section{REFERENCES}

Aarskog NK, Vedeler CA (2000) Real-time quantitative polymerase chain reaction. A new method that detects both the peripheral myelin protein 22 duplication in Charcot-Marie-Tooth type $1 \mathrm{~A}$ disease and the peripheral myelin protein 22 deletion in hereditary neuropathy with liability to pressure palsies. Hum Genet 107: 494-498.

Ammar N, Nelis E, Merlini L, Barisisc N, Amouri R, Ceuterick Ch, Martin JJ, Timmerman V, Hentati F, De Jonghe P (2003) Identification of novel GDAP1 mutations causing autosomal recessive Charcot-Marie-Tooth disease. Neuromuscular Disorders 13: 720-728.

Baxter RV, Ben Othmane K, Rochelle JM, Stajich JE, Hulett C, DewKnight S, Hentati F, Ben Hamida N, Bel S, Stengert JE, Gilbert J, Pericak-Vance M.A, Vance JM (2002) Ganglioside-induced differentiation associated-protein-1 is mutant in Charcot-Marie-Tooth disease type 4A/ 8q21. Nat Genet 30: 21-22.

Cassereau J, Chevrollier A, Bonneau D, Verny C, Procaccio V, Reynier P, Ferre M (2011) A locus-specific database for mutations in GDAP1 allows analysis of genotype-phenotype correlations in Charcot-Marie-Tooth diseases type $4 \mathrm{~A}$ and $2 \mathrm{~K}$. Orphanet J Rare Dis 6: 87. Claramunt R, Pedrola R, Sevilla T, de Maunain L, Berciano J, Cuesta A, Sanchez-Navarro B, Millan J.M, Saifi G, Lupski J. R, Vilchez J, Espinos C, Palau F (2005) Genetics of Charcot-Marie-Tooth disease type 4A: mutations, inheritance, phenotypic variability, and founder effect. I Med Genet 42: 358-365.

Cuesta A, Pedrola L, Sevilla. T, Garcia-Plannels J, Chumillas M. J, Mayordomo F, Le Guern E, Marin L, Vilchez JS, Palau F (2002) The gene encoding ganglioside-induced differentiation-associated protein 1 is mutated in axonal Charcot-Marie-Tooth type 4A disease. Nat Genet 30: 22-25.

Ionasescu VV (1995) Charcot-Marie-Tooth neuropathies: from clinical description to molecular genetics. Muscle Nerve 18: 267-275.

Kabzińska D, Pierscińska J, Kochański A (2009) Screening of the $17 \mathrm{p} 11.2-\mathrm{p} 12$ region in a large cohort of patients with Charcot-Marie-Tooth (CMT) disease or hereditary neuropathy with liability to pressure palsies (HNPP). J Appl Genet 50: 283-288.

Kabzinska D, Strugalska-Cynowska H, Kostera-Pruszczyk A, Ryniewicz B, Posmyk R, Midro A, Seemann P, Barankowa I, Zimon M, Baets J, Timmerman V, Guergueltcheva V, Yournev I, Sarafov S, De Jonghe P, Jordanova A, Hausmanowa-Petrusewicz I, Kochański A (2010) L239F founder mutation in GDAP1 is associated with a mild Charcot-Marie-Tooth type 4C4 (AR-CMT2C) phenotype. Neurogenetics 11: 357-366.

Niemann A, Ruegg M, La Padula V, Schenone A, Suter U. (2005) Ganglioside-induced differentiation associated protein 1 is a regulator of the mitochondrial network: new implications for CharcotMarie-Tooth disease. J Cell Biol 170: 1067-1078.

Niemann A, Wagner KM, Ruegg M, Suter U (2009) GDAP1 mutations differ in their effects on mitochondrial dynamics and apoptosis depending on the mode of inheritance. Neurobiol Dis 36: 509-520.

Rouzier C, Bannwarth S, Chaussenot A, Chevrollier A, Verschueren A, Bonello-Palot N, Fragaki K, Cano A, Pouget J, Pellissier JF, Procaccio V, Chabrol B, Paquis-Flucklinger V (2012) The MFN2 gene is responsible for mitochondrial DNA instability and optic atrophy 'plus' phenotype. Brain 135: 23-34.

Sivera R, Espinos C, Vilchez JJ, Mas F, Martinez-Rubio D, Chumillas MJ, Mayordomo F, Muelas N, Bataller I, Palau F, Sevilla T (2010) Phenotypical features of the p.R120W mutation in the GDAP1 
gene causing autosomal dominant Charcot-Marie-Tooth disease. J Periph Nerv Syst 15: 334-344.

Vital A, Latour P, Sole G, Ferrer X, Rouanet M, Tison F, Vital C, Goizet C (2012) A French family with Charcot-Marie-Tooth disease related to simultaneous heterozygous MFN2 and GDAP1 mutations. Neuromuscul Disord 22: 735-741.
Zimon M, Baets J, Fabrizi GM, Jakkolla E, Kabzinska D, Pilch J, Schindler AB, Cornblath DR, Fischbeck KH, Auer-Grumbach M, Guelly C, Huber N, De Vriendt E, Timmerman V, Suter U, Hausmanowa-Petrusewicz I, Niemann A, Kochanski A, De Jonghe P, Jordanova A (2011) Dominant GDAP1 mutations cause predominantly mild CMT phenotypes. Neurology 77: 540-548. 